\title{
Spinal Intradural Primary Malignant Peripheral Nerve Sheath Tumor with Leptomeningeal Seeding: Case Report and Literature Review
}

\author{
Humain BAHARVAHDAT ${ }^{1}$, Babak GANJEIFAR ${ }^{1}$, Nema Mohamadian ROSHAN ${ }^{2}$, Aslan BARADARAN ${ }^{3}$ \\ ${ }^{1}$ Division of Neurological Surgery, Ghaem Hospital, Mashhad University of Medical Sciences, Iran \\ ${ }^{2}$ Division of Pathology, Ghaem Hospital, Mashhad University of Medical Sciences, Iran \\ ${ }^{3}$ Orthopedic Research Center, Ghaem Hospital, Mashhad University of Medical Sciences, Iran
}

\section{ABSTRACT}

Spinal intradural primary malignant peripheral nerve sheath tumors (MPNST) are rare in patients without neurofibromatosis. Here we represent a 3-year-old girl of primary intradural spinal malignant peripheral nerve sheath tumor. The tumor was removed partially and MPNST was diagnosed in the histopathological examination. Her condition deteriorated due to acute hydrocephalus in the following days.

In this article, we discuss the clinical presentation, imaging, treatment, and prognosis of our patient and the other 22 patients of primary intradural MPNST, found in the literature. The Kaplan-Meier method was applied for univariate analysis and Cox proportional hazards model for multivariate analysis. This analysis showed that age, was an important factor predicting short-term survival of patients with MPNST.

KEYWORDS: Malignant peripheral nerve sheath tumor, Spinal intradural tumor, Hydrocephalus

ABBREVIATIONS: MPNST: Malignant peripheral nerve sheath tumor, NF-1: Neurofibromatosis type 1, MRI: Magnetic resonance imaging, T1WI: T1-weighted image, T2WI: T2-weighted image, CT: Computed tomography, ICU: Intensive care unit.

\section{INTRODUCTION}

$\mathrm{M}$ alignant peripheral nerve sheath tumor (MPNST) is a sarcoma arising from Schwann cells and neural crest cells (20). They constitute $5 \%$ of all soft tissue sarcomas with the incidence of $0.001 \%(10)$, and a peak incidence in the seventh decade of life (11); occurring more in patients with neurofibromatosis I (NF-1) (13). MPNSTs are usually located in extremities, trunk, and head and neck regions in order of decreasing frequency (19). Primary spinal intradural MPNSTs are extremely rare $(1,17,22,23)$, with frequent association with NF-1 (9).

Here, we presented an exceedingly rare case of MPNST diagnosed in a 3-year-old girl, with intradural extra-medullary involvement of the cervical spinal cord and diffuse leptomeningeal metastasis. We also reviewed the literature to discuss the management strategies and prognosis of primary spinal MPNST. We performed the systematic search by two researchers independently conducting the initial identification, and abstract screening. The eligible studies were discussed and the disagreements were resolved by means of discussion. The search was conducted on March 2015 in MedLine and EMBASE databases. For primary identification of articles, we searched the phrase "[(malignant) AND (peripher*) AND (nerv*) AND (tumor)]" setting no limitations, and if the abstract was not available, full text of article was used for screening evaluation. We assessed all studies regarding "the malignant peripheral nervous sheath tumors" located or involving the spinal canal. 
We included only studies with survival description of the patients. We reviewed all eligible cases for their clinical characteristics, management approaches. We excluded neurofibromatosis patients, and articles that the required data could not be extracted from the published or raw data asked from the corresponding author. Duplicate studies were discarded and only the most recent studies of each group were included. Survival curves were calculated by the Kaplan-Meier method and statistical differences were determined by the log-rank test. $P$ values less than 0.05 are considered significant. No corrections for multiple testing are considered. Analyses have been performed using SPSS software (version 16.0).

\section{CASE REPORT}

A 3-year-old girl was admitted to our hospital in September 2013, complaining of being unable to walk and talking. She had a history of severe low-back pain, gait disturbance, and progressive quadriparesis for one month. She had no past medical history or family history for any neoplastic diseases or neurofibromatosis. There were no superficial stigmata of neurofibromatosis. Motor deficit was noted in upper extremities with grades $2 / 5$ for both shoulders and elbows, and with grade 0/5 for both hands. She had spastic paraplegia.

Magnetic resonance imaging (MRI) revealed an intradural extra-intramedullary lesion in the cervical spinal canal from C1 to $\mathrm{T} 1$, which was isointense on T1-weighted image (T1WI), and heterogenously hyperintense on T2-weighted image (T2WI) (Figure 1). The lesion was enhanced nonhomogenously in MRI. Also there were multiple intradural extramedullary lesions in dorsal and lumbar parts of the spinal cord. They were mostly hypointense to cord in T2WI and isointense in T1WI (Figure 1). These lesions were enhanced homogenously. Pathologic thickening and leptomeningeal enhancement were noted along the spinal canal. Computed tomography (CT) scans of the chest, abdomen, and pelvic showed no abnormalities. The brain MRI was performed, revealing three small enhancing lesions in brainstem and temporal lobe.

Following laminectomy from $\mathrm{C} 1$ to $\mathrm{C} 6$, a longitudinal incision was made on the center of the exposed dura mater. A darkred large mass lesion was noted from $C 1$ to $C 7$ that invaded the spinal cord leading to its enlargement. The mass lesion also involved C2-C3 spinal roots. The extra-medullary part was first decompressed. Then the spinal cord was cut in posterior midline aspect for removing the intra-medullary part of tumor. Only partial excision was performed due to severe invasion of the spinal cord.

Microscopic examination showed varied cellularity with diffuse spindle cell proliferation and extensive necrosis. Proliferation of neoplastic cells with pleomorphic and hyperchromatic round to spindle cell nuclei was seen (Figures 2, 3). On immunohistological examinations: malignant cells expressed vimentin, S-100 protein and CD99, but not glial fibrillary acidic protein, epithelial membrane antigen, human melanin antigen 45 , or synaptophysin. Histopathological diagnosis was compatible with malignant nerve sheath tumor.
After surgery, the patient was admitted to the neurosurgical intensive care unit (ICU) with worsening of her right hand deficit. The genomic test for NF1 mutation came as negative. The patient had an uneventful period until day 9 when she experienced loss of consciousness with both nonreactive mydriasis. Axial brain CT scan revealed severe hydrocephalus due to obstruction in the lower brain stem region (Figure 4). Despite insertion of external ventricular drainage and aggressive medical treatment she did not improve and died.

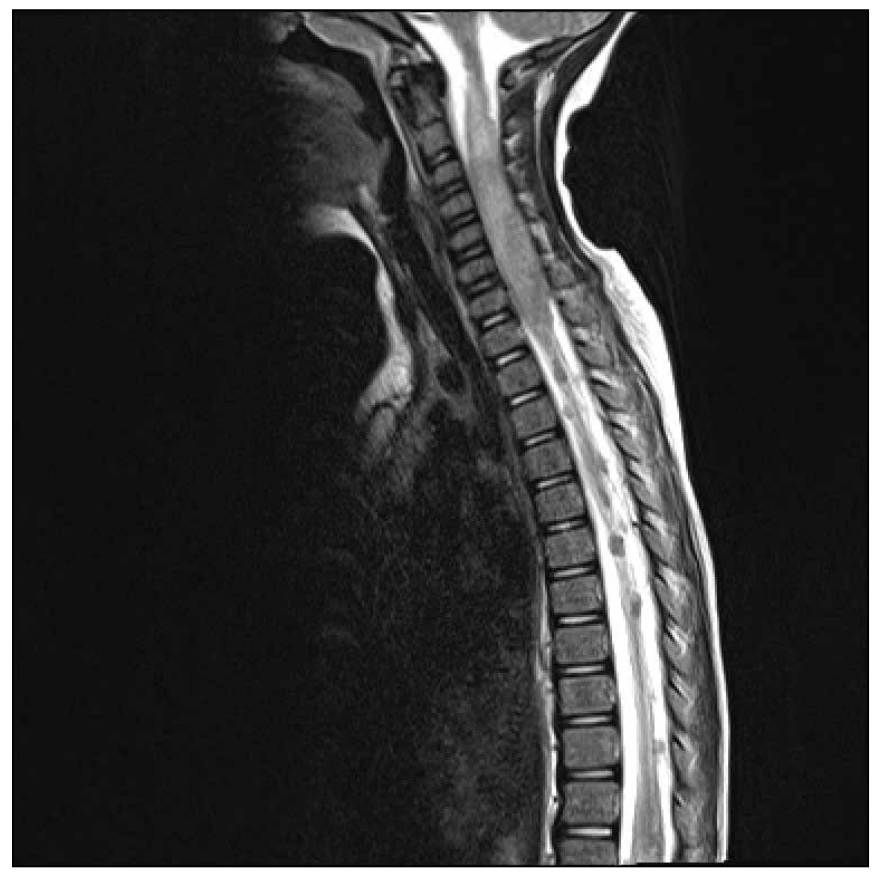

Figure 1: Magnetic resonance imaging (MRI) revealed an intradural extra-intramedullary lesion in the cervical spinal canal from $\mathrm{C} 1$ to $\mathrm{T} 1$, heterogenous on T2-weighted image (T2WI) and associated with multiple intradural extramedullary drop metastases.

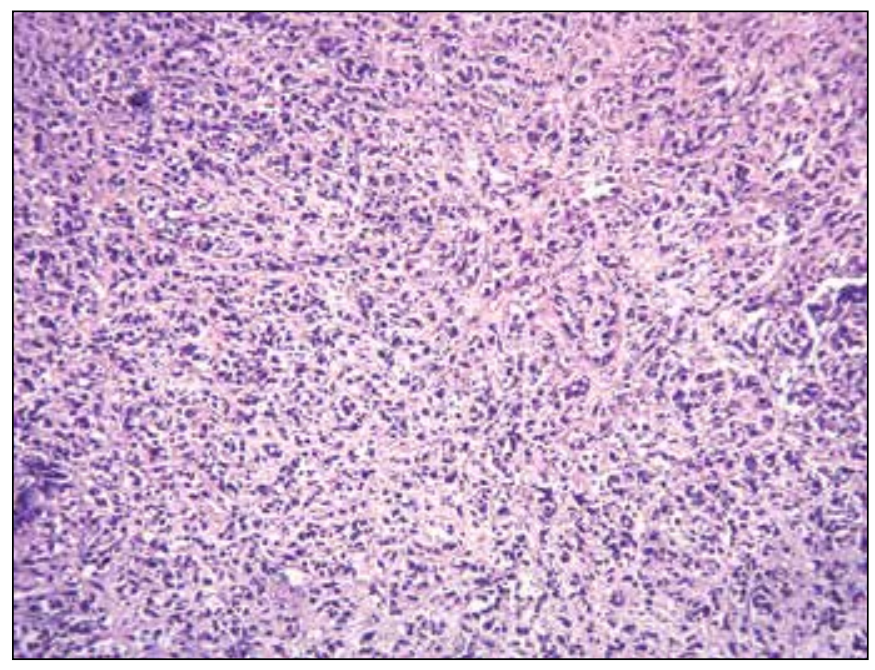

Figure 2: Proliferation of neoplastic cells with pleomorphic and hyperchromatic round to spindle cell nuclei. (H\&E, x100). 


\section{DISCUSSION}

Intradural spinal MPNSTs can be classified as primary or metastatic. (6). Primary spinal intradural MPNST is extremely rare. We found only 22 cases of primary spinal MPNST without neurofibromatosis in our search to this date. We summarized them in Table I. They are primarily distributed in: cervical in 7 patients $(30 \%)(2,8,17,23)$, thoracic and thoracolumbar in $6(26 \%)(7,15,17,23)$, and lumbar in 10 $(44 \%)(1,3,7,14,17,21,22,24,25)$. Our patient had an intradural extramedullary cervical tumor extending from foramen magnum to lower cervical vertebrae with severely invasion of the spinal cord.

As shown in Table I, most patients of primary spinal MPNST were young with mean age of $32 \pm 19$ (SD) years and range of 3-70 years. There are only 5 children (22\%) in the reports $(8,17,24,25)$, mostly under 10 years old. Our patient is the

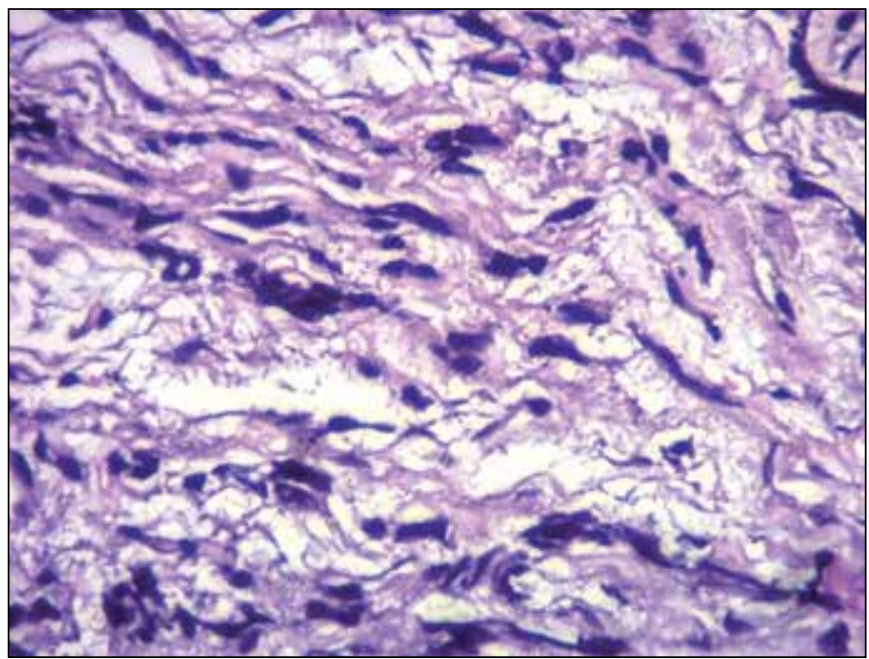

Figure 3: Wavy nuclei with hyperchromasia and pleomorphism. (H\&E, x400).

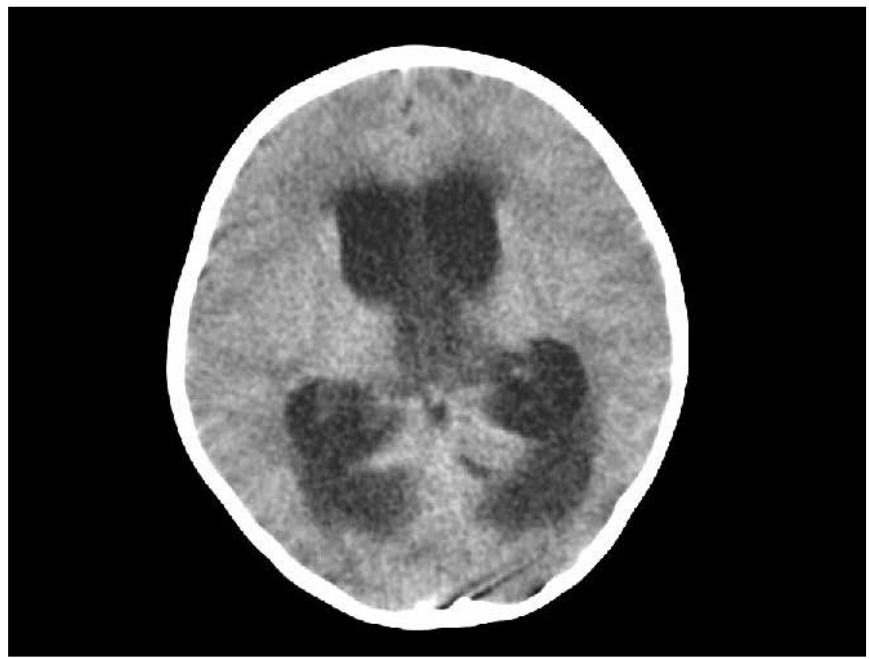

Figure 4: CT scan showed severe hydrocephalus after the patient's loss of consciousness. youngest one. Tumors are distributed equally between men and women.

Patients usually present with pain, weakness, sensory loss, and bladder/bowel deficits. Hydrocephalus was seen in 4 patients with spinal MPNSTs $(8,14,15)$, including 3 patients with brain metastasis and one with the cervical spinal cord involvement. Our patient developed acute hydrocephalus during hospitalization. Prompt diagnosis of acute hydrocephalus can prevent morbidity and mortality.

As found in our patient, the spinal MPNST lesions were described as non-homogenous hyperintense in T2WI and isointense in $\mathrm{T} 1 \mathrm{WI}$ that enhanced after gadolinium injection $(15,21)$. The hyperintense area on T2Wl was reported as a central cystic lesion or necrosis (4). The leptomeningeal metastasis was hypointense in T2WI and enhanced homogenously $(14,15,21,24)$. The spinal seeding is defined as a "string-of-beads" pattern since they are small enhancing lesions spreading along the spinal cord (15). MRI may reveal leptomeningeal thickening and enhancement (21).

Pathological characteristics of spinal MPNST are high cellularity with spindle-shaped cells, nuclear atypia, increased mitotic activity, necrosis, extreme nuclear anaplasia, and endothelial proliferation $(15,23)$.

Surgery is currently the mainstay of treatment for intradural spinal MPNST. It can result in significant recovery with an improved prognosis $(1,10,16,23)$. Total removal was achieved in $64 \%$ of the patients in the previous studies (Table I). We could not achieve complete removal of the tumor, given that the lesion severely invaded the spinal cord. To the best of our knowledge, this is the only reported case with such massive tumor invasion into the spinal cord. The best adjuvant treatment for spinal MPNSTs remains poorly defined due to the rarity of the tumor and the lack of prospective trials.

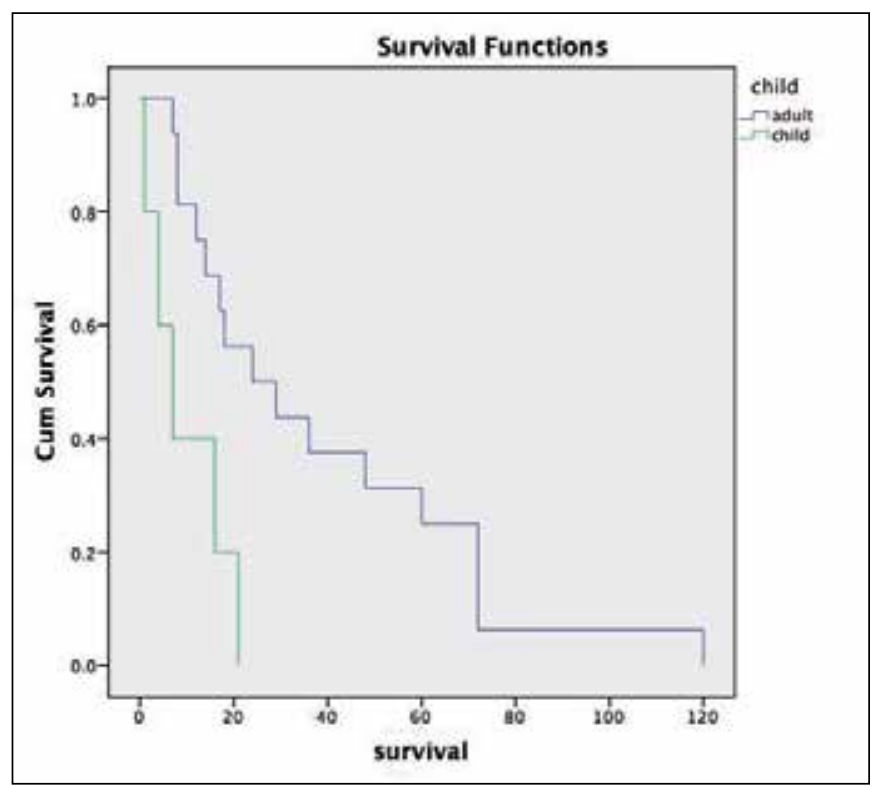

Figure 5: Kaplan-Meier test curve, comparing the survival rates between children ( $<16$ years) and adults. 


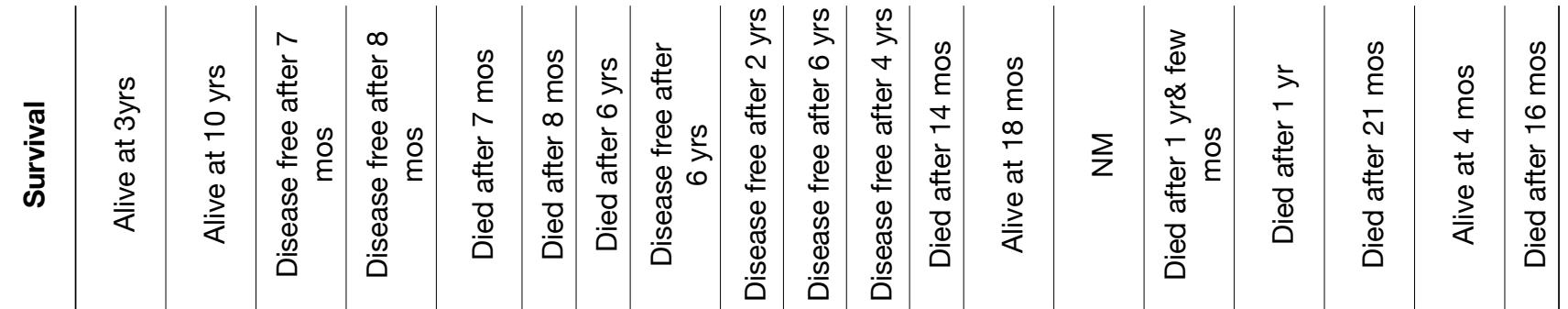

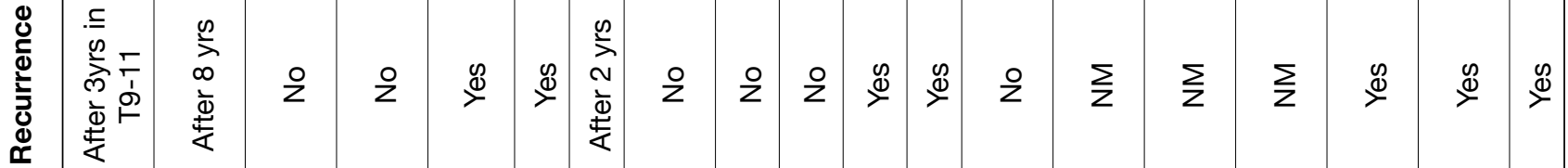

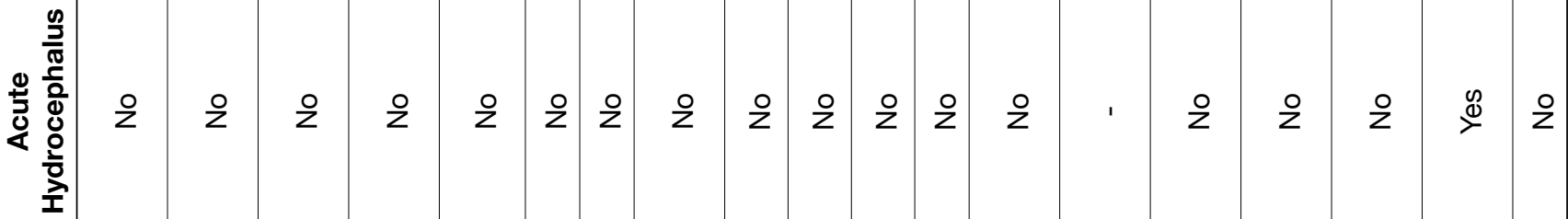

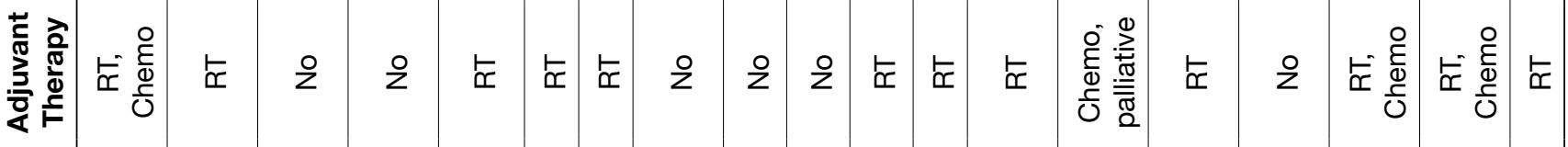

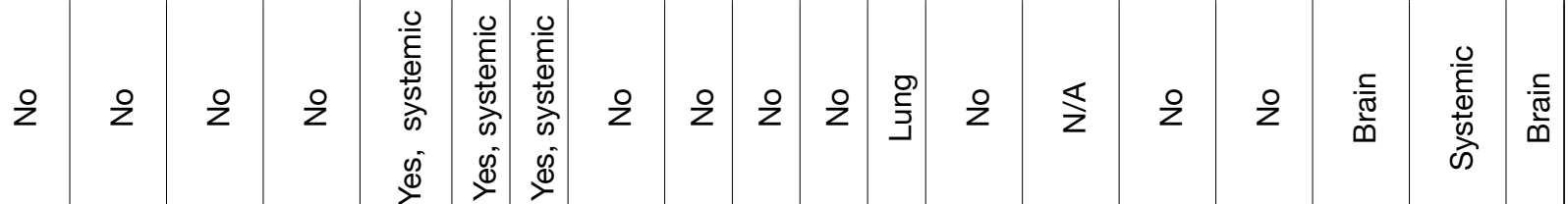

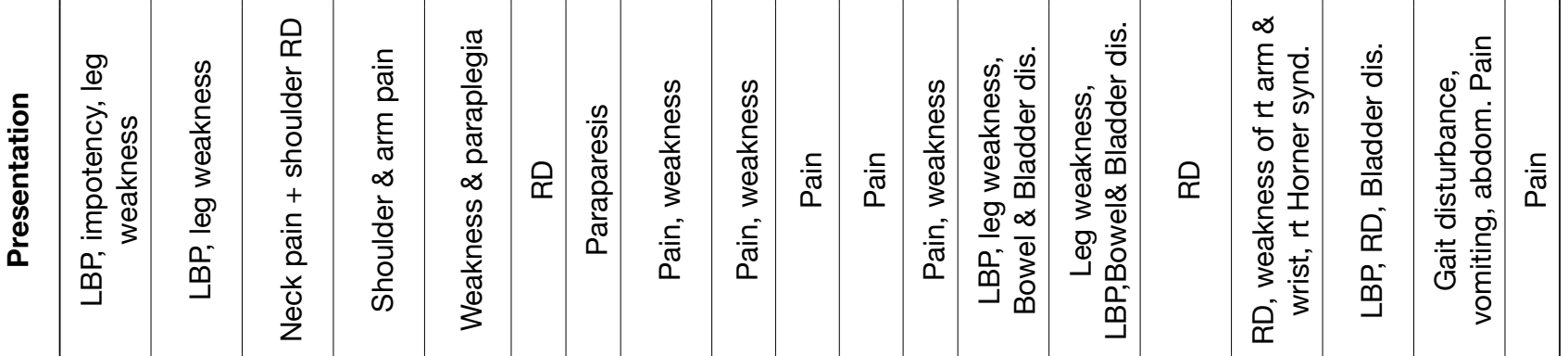

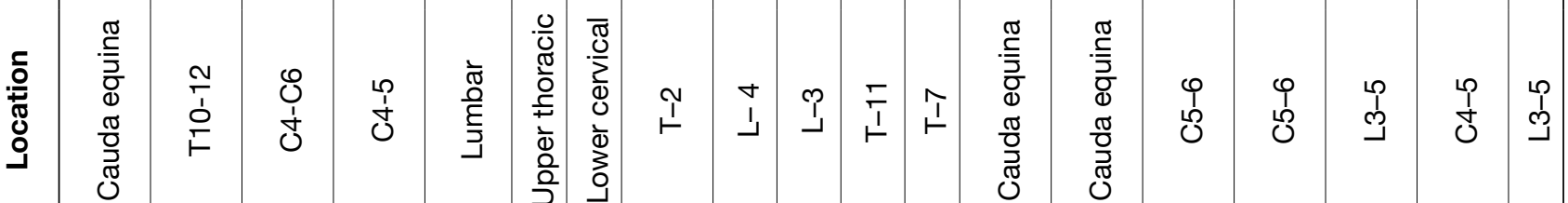

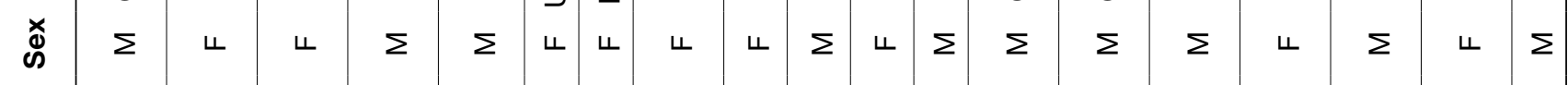

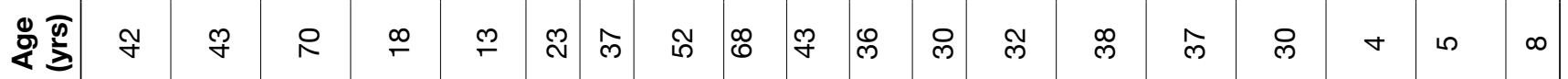
¿

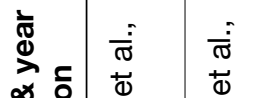

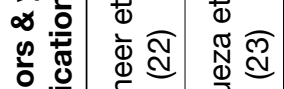

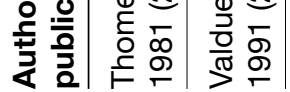

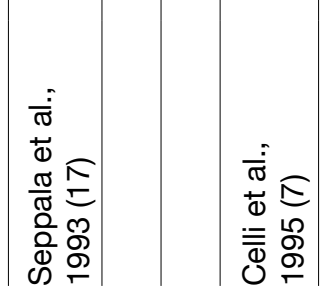

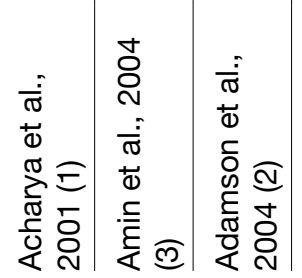

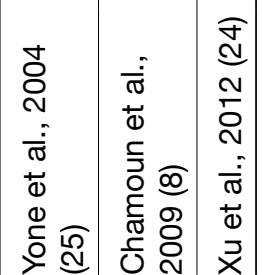


Radiation therapy has been beneficial for local tumor control especially when complete resection is impossible $(1,3)$. Yet, the effectiveness of chemotherapy has not been proven for MPNST (5).

Prognosis of patients with MPNST has always been poor with a 5 -year survival rate of $16 \%$ to $52 \%$ (12). The 1 -year and 5 -year survival rate in our review of primary spinal MPNSTs was $68 \%$ $(15 / 22)$ and $23 \%$ (5/22), respectively. Even after complete removal of the tumor and radiation therapy, metastasis rate was $45 \%$ (10/22), with recurrence of $54 \%$ (12/22). In these 23 reported spinal MPNSTs, we found age as a prognostic factor, showing children surviving less than adults $(p=0.005$, Figure 5). But the primary location of tumor (cervical, dorsal, or lumbar), sex (male or female), resection of the tumor (total or partial), occurrence of hydrocephalus, presence of metastasis, recurrence, or radiotherapy did not have any significant effect on the survival of patients $(p>0.05)$.

Craniospinal metastasis was reported in 6 cases of primary intradural spinal MPNST $(14,15,18,21,24)$, including our patient. In 2 patients, leptomeningeal seeding was present at the time of diagnosis (21). Our patient was the only child who had metastasis along the whole spinal cord. Leptomeningeal spread is a dreadful and fatal event, usually suggesting advanced malignant disease.

\section{CONCLUSION}

Primary spinal MPNST have a high predisposition to leptomeningeal, craniospinal, and systemic metastasis with a rare incidence of spinal cord invasion. Despite aggressive treatment, its mortality and morbidity rates are high. Age may play a role in determining the prognosis of these patients, but one should bear in mind that this analysis was conducted on a limited number of patients reported in the literature. Lack of statistical significance for other variables could also be due to this matter.

\section{REFERENCES}

1. Acharya $R$, Bhalla $S$, Sehgal $A D$ : Malignant peripheral nerve sheath tumor of the cauda equina. Neurol Sci 22:267-270, 2001

2. Adamson DC, Cummings TJ, Friedman AH: Malignant peripheral nerve sheath tumor of the spine after radiation therapy for Hodgkin's lymphoma. Clin Neuropathol 23:245255, 2004

3. Amin A, Saifuddin A, Flanagan A, Patterson D, Lehovsky J: Radiotherapy-induced malignant peripheral nerve sheath tumor of the cauda equina. Spine (Phila Pa 1976) 29:E506509, 2004

4. Aydin MD, Yildirim U, Gundogdu C, Dursun O, Uysal HH, Ozdikici M: Malignant peripheral nerve sheath tumor of the orbit: Case report and literature review. Skull Base 14:109113; discussion 113-104, 2004

5. Baehring JM, Betensky RA, Batchelor TT: Malignant peripheral nerve sheath tumor: The clinical spectrum and outcome of treatment. Neurology 61:696-698, 2003 
6. Baek WS, Pytel P, Undevia SD, Rubeiz H: Spinal cord metastasis of a non-neurofibromatosis type-1 malignant peripheral nerve sheath tumor: An unusual manifestation of a rare tumor. J Neurooncol 74:183-185, 2005

7. Celli P, Cervoni L, Tarantino R, Fortuna A: Primary spinal malignant schwannomas: Clinical and prognostic remarks. Acta Neurochir (Wien) 135:52-55, 1995

8. Chamoun RB, Whitehead WE, Dauser RC, Luerssen TG, Okcu MF, Adesina AM, Jean A: Primary disseminated intradural malignant peripheral nerve sheath tumor of the spine in a child: Case report and review of the literature. Pediatr Neurosurg 45:230-236, 2009

9. Dimou J, Jithoo R, Pitcher M, White G: Recurrent malignant peripheral nerve sheath tumour in a patient with neurofibromatosis Type 1: A case report. J Clin Neurosci 16:1221-1223, 2009

10. Gupta G, Maniker A: Malignant peripheral nerve sheath tumors. Neurosurg Focus 22:E12, 2007

11. Hajdu SI: Peripheral nerve sheath tumors. Histogenesis, classification, and prognosis. Cancer 72:3549-3552, 1993

12. Kourea HP, Bilsky MH, Leung DH, Lewis JJ, Woodruff JM: Subdiaphragmatic and intrathoracic paraspinal malignant peripheral nerve sheath tumors: A clinicopathologic study of 25 patients and 26 tumors. Cancer 82:2191-2203, 1998

13. Lai RS, Lin SL, Hsu SS, Wu MT: Intrathoracic paraspinal malignant peripheral nerve sheath tumor. J Chin Med Assoc 69:37-41, 2006

14. Lau D, Moon DH, Park P, Hervey-Jumper S, McKeever $\mathrm{PE}$, Orringer DA: Radiation-induced intradural malignant peripheral nerve sheath tumor of the cauda equina with diffuse leptomeningeal metastasis. J Neurosurg Spine 21:719-726, 2014

15. Li Y, Fan F, Xu J, An J, Zhang W: Primary malignant peripheral nerve sheath tumor of the spine with acute hydrocephalus: $A$ rare clinical entity. J Neurosurg Spine 21:367-371, 2014
16. Prieto R, Pascual JM, Garcia-Cabezas MA, Lopez-Barea F, Barrios L, Gonzalez-Llanos F: Low-grade malignant triton tumor in the lumbar spine: A rare variant of malignant peripheral nerve sheath tumor with rhabdomyoblastic differentiation. Neuropathology 32:180-189, 2012

17. Seppala MT, Haltia MJ: Spinal malignant nerve-sheath tumor or cellular schwannoma? A striking difference in prognosis. J Neurosurg 79:528-532, 1993

18. Stark AM, Mehdorn HM: Leptomeningeal metastasis of an intradural malignant peripheral nerve sheath tumor. $\mathrm{J}$ Clin Neurosci 20:1181-1183, 2013

19. Stucky CC, Johnson KN, Gray RJ, Pockaj BA, Ocal IT, Rose PS, Wasif N: Malignant peripheral nerve sheath tumors (MPNST): The Mayo Clinic experience. Ann Surg Oncol 19:878-885, 2012

20. Than KD, Ghori AK, Wang AC, Pandey AS: Metastatic malignant peripheral nerve sheath tumor of the cauda equina. J Clin Neurosci 18:844-846, 2011

21. Thomas JG, Lincoln C, Clay Goodman J, Gopinath SP: Malignant peripheral nerve sheath tumor of the cauda equina with craniospinal metastasis. J Clin Neurosci 21:2239-2242, 2014

22. Thomeer RT, Bots GT, van Dulken H, Luyendijk W, Helle P: Neurofibrosarcoma of the cauda equina. Case report. J Neurosurg 54:409-411, 1981

23. Valdueza JM, Hagel C, Westphal M, Hansel M, Herrmann HD: Primary spinal malignant schwannoma: Clinical, histological and cytogenetic findings. Neurosurg Rev 14:283-291, 1991

24. Xu Q, Xing B, Huang X, Wang R, Li Y, Yang Z: Primary malignant peripheral nerve sheath tumor of the cauda equina with metastasis to the brain in a child: Case report and literature review. Spine J 12:e7-13, 2012

25. Yone K, ljiri K, Hayashi K, Yokouchi M, Takenouchi T, Manago $\mathrm{K}$, Nerome $\mathrm{Y}$, ljichi O, Ikarimoto $\mathrm{N}$, Komiya S: Primary malignant peripheral nerve sheath tumor of the cauda equina in a child case report. Spinal Cord 42:199-203, 2004 